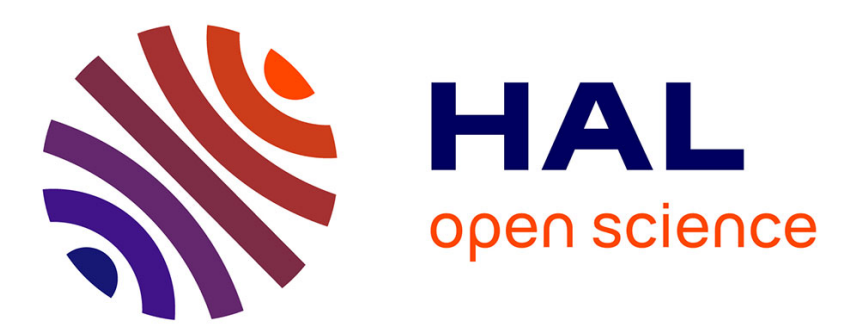

\title{
Boundary element method for 3D conductive thin layer in eddy current problems
}

Mohammad Issa, Jean-René Poirier, Olivier Chadebec, Victor Péron, Ronan

Perrussel

\section{- To cite this version:}

Mohammad Issa, Jean-René Poirier, Olivier Chadebec, Victor Péron, Ronan Perrussel. Boundary element method for 3D conductive thin layer in eddy current problems. COMPEL: The International Journal for Computation and Mathematics in Electrical and Electronic Engineering, 2019, 38 (2), pp.502-521. 10.1108/COMPEL-09-2018-0348 . hal-02320511

\section{HAL Id: hal-02320511 \\ https://hal.inria.fr/hal-02320511}

Submitted on 5 Nov 2019

HAL is a multi-disciplinary open access archive for the deposit and dissemination of scientific research documents, whether they are published or not. The documents may come from teaching and research institutions in France or abroad, or from public or private research centers.
L'archive ouverte pluridisciplinaire HAL, est destinée au dépôt et à la diffusion de documents scientifiques de niveau recherche, publiés ou non, émanant des établissements d'enseignement et de recherche français ou étrangers, des laboratoires publics ou privés. 


\title{
Boundary Element Method for 3D Conductive Thin Layer in Eddy Current Problems
}

\author{
Mohammad ISSA, Jean-René POIRIER, Olivier CHADEBEC, Victor PÉRON, and \\ Ronan PERRUSSEL
}

\begin{abstract}
Purpose - Thin conducting sheets are used in many electric and electronic devices. Solving numerically the eddy current problems in presence of these thin conductive sheets requires a very fine mesh which leads to a large system of equations, and it becomes more problematic in case of higher frequencies. The purpose of this paper is to show the numerical pertinence of equivalent models for $3 \mathrm{D}$ eddy current problems with a conductive thin layer of small thickness $e$ based on the replacement of the thin layer by its mid-surface with equivalent transmission conditions that satisfy the shielding purpose, and by using an efficient discretization using the Boundary Element Method (BEM) in order to reduce the computational work.

Design/methodology/approach - These models are solved numerically using the BEM and some numerical experiments are performed to assess the accuracy of our models. The results are validated by comparison with an analytical solution and a numerical solution by the commercial software Comsol.

Findings - The error between the equivalent models and the analytical and numerical solutions confirms the theoretical approach. In addition to this accuracy, the time consumption is reduced by considering a discretization method that requires only a surface mesh.

Originality/Value - Based on an hybrid formulation, we present briefly a formal derivation of impedance transmission conditions for 3D thin layers in eddy current problems where non-conductive materials are considered in the interior and the exterior domain of the sheet. BEM is adopted to discretize the problem as there is no need for a volume discretization.
\end{abstract}

Keywords - Eddy Current Problems, Thin Conducting Layers, Transmission Conditions, Boundary Element Method

\section{Introduction}

Many magnetic components are surrounded by conductive thin layers for shielding purpose. Modelling these conducting regions requires very fine volume discretizations because the fields decay rapidly through the surface due to the skin depth. Also, it leads to a large system of equations and then to a prohibitive computational time especially for 3D structures. To overcome this difficulty, the conductive sheet can be replaced by a mid-surface with equivalent transmission conditions. These transmission conditions can be derived asymptotically for vanishing sheet thickness $e$ where the skin depth is kept less than or equal to the thickness $e$, see e.g. (K. Schmidt and A. Chernov, 2013, 2014; Victor Péron, 2017; Victor Péron, Kersten Schmidt, Marc Duruflé, 2016).

In (K. Schmidt and A. Chernov, 2014) two families of Transmission Conditions for Eddy Current Model 
in 2D have been derived using asymptotic expansions, ITC-1-N based on scaling the conductivity with the sheet thickness $e$ like 1/e and ITC-2-N based on scaling the conductivity with the sheet thickness $e$ like $1 / e^{2}$, where $N+1$ is the order of convergence for these families. The robustness of the ITC-2-N family of transmission conditions is validated in (K. Schmidt and A. Chernov, 2013) and it shows a higher accuracy in comparison with ITC-1-N. The ITC-2-N family thus adopted in (Victor Péron, Kersten Schmidt, Marc Duruflé, 2016) to derive an equivalent transmission conditions for the full time-harmonic Maxwell equations in 3D, where curved thin sheets are considered, and the material constants can take different values inside and outside the sheet.

In this work, the family ITC-2-N is considered, We present briefly a formal derivation of impedance transmission conditions for 3D eddy current problems, where curved thin sheets are considered, and the materials inside and outside the sheet are non-conductive. There are several differences between this work and the work in Ref. (Victor Péron, 2017). First, the work in (Victor Péron, 2017) is concerned essentially with theoretical objectives (well-posedness, stability and convergence results for the asymptotic models) whereas this work is concerned essentially with numerical objectives. Second, in (Victor Péron, 2017), the derivation of asymptotic models is based on a magnetic field formulation and a multi-scale expansion for the magnetic field and then impedance conditions are identified for the electric field, whereas, in this work, we proceed with an hybrid (electric and magnetic fields) formulation.

We also study a discretization that can be the numerical relevant for ITCs. We avoid the volume mesh required in many numerical methods, e.g. in the Finite Element Method (FEM), by discretizing the problem using the Boundary Element Method (BEM) that uses only a mesh on the surface (S. Rjasanow, O. Steinbach, 2007). In addition, BEM is well adapted to general field problems with unbounded structures because no artificial boundaries are needed, this is not the case for FEM. The BEM becomes a powerful tool for numerical studies in computational electromagnetism, In (J.M. Schneider and S.J. Salon, 1980) BEM has been used in some applications for eddy current problems based on the electric vector potential formulation. Using the same formulation, a BEM is developped for calculating induced eddy current flows in conducting

plates with cracks in (M. Morjaria, S. Mukherjee, F.C. Moons, 1982). A direct boundary element method for the computation of eddy currents in a linear homogeneous conductor is presented in (R. Hiptmair and $\mathrm{J}$. Ostrowski, 2005).

\subsection{Eddy Current Approximation of Maxwell equations}

We denote by $\Omega \subset \mathbb{R}^{3}$ the domain of study, which is itself composed of three sub-domains

$$
\Omega=\Omega_{-}^{e} \cup \overline{\Omega_{0}^{e}} \cup \Omega_{+}^{e}
$$

where $\Omega_{-}^{e}$ is the interior domain that corresponds to any non-conductive linear material, $\Omega_{+}^{e}$ is the exterior domain (air), and the subdomain $\Omega_{0}^{e}$ is a conductive thin layer of constant thickness $e$ surrounding the subdomain $\Omega_{-}^{e}$. Let $\Gamma_{-}^{e}$ and $\Gamma_{+}^{e}$ be the two smooth boundaries of the subdomains $\Omega_{-}^{e}$ and $\overline{\Omega_{-}^{e}} \cup \Omega_{0}^{e}$ respectively. And let $\Gamma$ be the mid-surface of the thin layer $\Omega_{0}^{e}$ (see Figure 1).

As the three subdomains have different properties, we define $\mu^{e}$ (magnetic permeability) and $\sigma^{e}$ (conductivity) as piecewise constant functions

$$
\mu^{e}=\left\{\begin{array}{lll}
\mu_{-} & \text {in } & \Omega_{-}^{e}, \\
\mu_{0}^{c} & \text { in } & \Omega_{0}^{e}, \\
\mu_{+} & \text {in } & \Omega_{+}^{e},
\end{array} \quad \text { and } \quad \sigma^{e}=\left\{\begin{array}{lll}
0 & \text { in } & \Omega_{-}^{e}, \\
\sigma_{0} & \text { in } & \Omega_{0}^{e}, \\
0 & \text { in } & \Omega_{+}^{e} .
\end{array}\right.\right.
$$




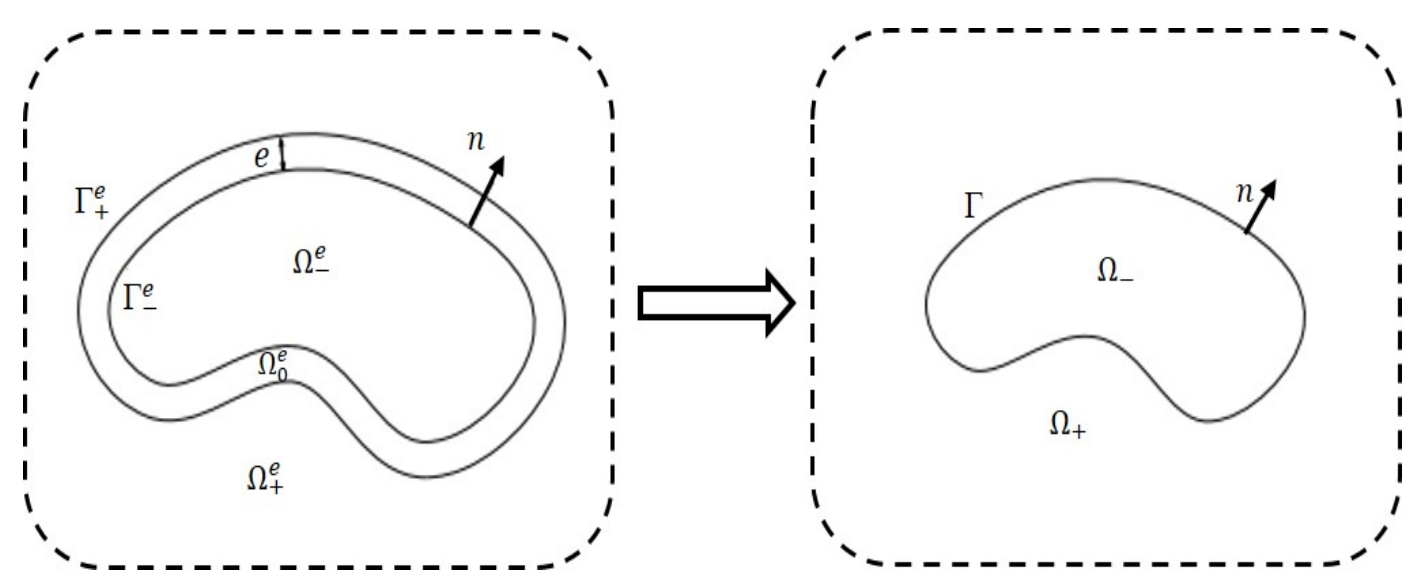

Figure 1: A cross section of the domain $\Omega$

We are interested in the case where the skin depth $\delta=\sqrt{\frac{2}{\omega \mu_{0}^{c} \sigma_{0}}}$ is smaller than $e$ or of the same order. For that, we assume an explicit dependence of the layer conductivity $\sigma_{0}$ on $e$

$$
\sigma_{0}=e^{-2} \bar{\sigma},
$$

which comes from the fact that, in the asymptotic limit, as the layer is thinner, the conductivity is larger and $\delta$ remains less than or equal to $e$. For simplicity, we assume that the source current term $J_{0}$ is smooth enough and its support does not meet the layer $\Omega_{0}^{e}\left(\boldsymbol{J}_{\mathbf{0}}=\mathbf{0}\right.$ in $\left.\boldsymbol{\Omega}_{\mathbf{0}}^{\boldsymbol{e}}\right)$, and we denote $\boldsymbol{J}^{ \pm}=J_{0}$ in $\Omega_{ \pm}^{e}$. Let us now recall the general model of the eddy current problem (Ana Alonso Rodriguez, and Alberto Valli, 2010)

$$
\begin{aligned}
\operatorname{curl} H^{e} & =J & & \text { in } \Omega, \\
\operatorname{curl} E^{e}-i \omega \mu^{e} H^{e} & =0 & & \text { in } \Omega, \\
B^{e} & =\mu^{e} H^{e} & & \text { in } \Omega, \\
J & =\sigma^{e} E^{e}+J_{0} & & \text { in } \Omega, \\
\operatorname{div} E^{e} & =0 & & \text { in } \Omega_{ \pm}^{e}, \\
\int_{\Gamma_{ \pm}^{e}} E_{ \pm}^{e} \cdot n d S & =0 & & \text { on } \Gamma_{ \pm}^{e}, \\
E^{e} & =O\left(\frac{1}{|x|}\right) & & \text { as }|x| \rightarrow \infty,
\end{aligned}
$$

where $\omega$ is the angular frequency.

The paper is organized as follows. Section 2 presents the hybrid formulation of the eddy current problem, the procedure for deriving the transmission conditions, and the equivalent models up to order 2 . In section 3 we provide the Boundary Element Method with special basis functions to solve the problem. The integral equations, variational formulations, and Galerkin discretisations are given for the terms of the expansion and the equivalent models. Numerical results are provided in section 4, we validate our models and assumptions, and we study the computational time using the BEM. Details about the calculation of the analytical solution and the postprocessing to calculate the external magnetic field are given in the Appendix. 


\section{Transmission Conditions}

In section 2.1, we state the hybrid formulation which is considered as a problem of departure in order to find the equivalent models presented in section 2.2. In the same section, we present briefly the multiscale expansion and the steps to derive the transmission conditions.

\section{$2.1 \quad \mathbf{E}_{ \pm}^{\mathrm{e}} / \mathbf{H}_{\mathbf{0}}^{\mathrm{e}}$ Hybrid Formulation}

In (Victor Péron, 2017) the formulations in $H$ and $E$ are adopted but that it is also possible to do all the calculations in a hybrid formulation. Let $u$ be a vector field on $\Gamma$, we denote by $[u]$ and $\{u\}$ respectively the jump and mean of $u$ across $\Gamma$

$$
[u]_{\Gamma}=u_{\Gamma_{\Gamma^{+}}}-u_{\Gamma_{\Gamma^{-}}} \text {, and }\{u\}_{\Gamma}=\frac{1}{2}\left(u_{\Gamma_{\Gamma^{+}}}+u_{\left.\right|_{\Gamma^{-}}}\right) .
$$

An $E$-based formulation is considered in the air region $\Omega_{ \pm}^{e}$ which reads

$$
\operatorname{curlcurl} E_{ \pm}^{e}=i \omega \mu_{ \pm}^{e} J_{0} .
$$

And an $H$-based formulation is considered in the conductor $\Omega_{0}^{e}$

$$
\text { curlcurl } H_{0}^{e}-i \omega \mu_{0}^{e} \sigma_{0}^{e} H_{0}^{e}=0 .
$$

The continuity of the tangential component of the magnetic field and the normal component of the induction field across the two conductor surfaces $\Gamma_{+}^{e}$ and $\Gamma_{-}^{e}$ are considered

$$
\begin{array}{ll}
{\left[H^{e} \times n\right]_{\Gamma_{ \pm}^{e}}=0} & \text { on } \Gamma_{ \pm}^{e}, \\
{\left[\mu H^{e} \cdot n\right]_{\Gamma_{ \pm}^{e}}=0} & \text { on } \Gamma_{ \pm}^{e} .
\end{array}
$$

Finally, the hybrid eddy current model can be reduced to the following

$$
\left\{\begin{array}{lll}
\operatorname{curlcurl} E_{ \pm}^{e} & =i \omega \mu_{ \pm}^{e} J_{0} & \text { in } \Omega_{ \pm}^{e}, \\
\operatorname{curlcurl} H_{0}^{e}-\left(k_{0}^{e}\right)^{2} H_{0}^{e} & =0 & \text { in } \Omega_{0}^{e}, \\
i \omega\left(H_{0}^{e} \times n\right) & =\frac{1}{\mu_{ \pm}^{e}} \operatorname{curl} E_{ \pm}^{e} \times n & \text { on } \Gamma_{ \pm}^{e}, \\
\mu_{0}^{c} H_{0}^{e} \cdot n & =\frac{1}{i \omega} \operatorname{curl} E_{ \pm}^{e} \cdot n & \text { on } \Gamma_{ \pm}^{e}, \\
\operatorname{div} E_{ \pm}^{e} & =0 & \text { in } \Omega_{ \pm}^{e}, \\
\int_{\Gamma_{ \pm}^{e}} E_{ \pm}^{e} \cdot n d S & =0 & \text { on } \Gamma_{ \pm}^{e}, \\
E^{e} & =O\left(\frac{1}{|x|}\right) & \text { as }|x| \rightarrow \infty,
\end{array}\right.
$$

where $k_{0}^{e}$ is the complex wave number given by

$$
\left(k_{0}^{e}\right)^{2}(x)=i \omega \sigma_{0}^{e}(x) \mu_{0}^{e}(x),
$$

$E_{+}^{e}, E_{-}^{e}$ and $H_{0}^{e}$ denote the restriction of $E^{e}$ and $H^{e}$ to the respective domains $\Omega_{+}^{e}, \Omega_{-}^{e}$ and $\Omega_{0}^{e}$.

\subsection{Multiscale Expansion and Equivalent Models with Transmission Conditions}

We propose replacing the thin layer by its mid-surface $\Gamma$, on which appropriate conditions are set. Therefore, we have to approximate new models defined in $e$-independent domains $\Omega_{-}$and $\Omega_{+}$(see Figure 1 ) where

$$
\Omega_{-}=\lim _{e \rightarrow 0} \Omega_{-}^{e}, \quad \text { and } \quad \Omega_{+}=\lim _{e \rightarrow 0} \Omega_{+}^{e} .
$$


In the approximate model and structure, we can redefine the magnetic properties in the new subdomains by a simple extension of $\mu^{e}$ and $\sigma^{e}$ outside the sheet. To obtain the new values:

$$
\mu=\left\{\begin{array}{lll}
\mu_{-} & \text {in } \Omega_{-}, \\
\mu_{+} & \text {in } \Omega_{+},
\end{array} \quad \text { and } \sigma=\left\{\begin{array}{lll}
\sigma_{-}=0 & \text { in } \Omega_{-}, \\
\sigma_{+}=0 & \text { in } \Omega_{+} .
\end{array}\right.\right.
$$

\subsubsection{Steps to derive the Transmission Conditions}

Assuming that $\Gamma$ is a smooth surface, it is possible to derive a multiscale expansion for the solution of the problem: It possesses an asymptotic expansion in power series of the small parameter $e$.

$$
\begin{gathered}
E_{ \pm}^{e}(x) \approx E_{0}^{ \pm}(x)+e E_{1}^{ \pm}(x)+e^{2} E_{2}^{ \pm}(x)+\ldots+\mathscr{O}\left(e^{k}\right), \\
H_{0}^{e}(x) \approx \mathscr{H}_{0}\left(y_{\alpha}, \frac{h}{e}\right)+e \mathscr{H}_{1}\left(y_{\alpha}, \frac{h}{e}\right)+\ldots+\mathscr{O}\left(e^{k}\right),
\end{gathered}
$$

where $\mathscr{O}\left(e^{k}\right)$ means the remainder is uniformly bounded by $e^{k}$.

Here, $x \in \mathbb{R}^{3}$ are the cartesian coordinates, and $\left(y_{\alpha}, h\right)$ is the local normal coordinate system where $h \in\left(-\frac{e}{2}, \frac{e}{2}\right)$ and $y_{\alpha}$ for $\alpha=1,2$ are the normal and tangential coordinates to $\Gamma$, respectively.

The term $\mathscr{H}_{j}$ is a profile defined on $\Gamma \times\left(-\frac{1}{2}, \frac{1}{2}\right)$ and is smooth in all variables.

The derivation is based on (Victor Péron, 2017):

1. The expansion of the differential operators inside the thin layer $\Omega_{0}^{e}$

$$
L\left(y_{\alpha}, h ; D_{\alpha}, \partial_{3}^{h}\right)=\text { curlcurl }-\left(k_{0}^{e}\right)^{2} \mathbb{I} \quad \text { in } \Omega_{0}^{e} .
$$

2. The Taylor expansion of the component $\left.E_{j}\right|_{\Gamma_{ \pm}^{e}}$ of the expansion (2.8) around the mid-surface $\Gamma$

$$
\begin{array}{r}
\operatorname{curl} E_{j}^{ \pm} \times\left. n\right|_{h= \pm \frac{e}{2}}=\operatorname{curl} E_{j} \times\left. n\right|_{0 \pm} \pm \frac{e}{2} \partial_{h} \operatorname{curl} E_{j} \times\left. n\right|_{0 \pm}+\ldots \\
\left.\quad \operatorname{curl} E_{j}^{ \pm} \cdot n\right|_{h= \pm \frac{e}{2}}=\left.\operatorname{curl} E_{j} \cdot n\right|_{0 \pm} \pm\left.\frac{e}{2} \partial_{h} \operatorname{curl} E_{j} \cdot n\right|_{0 \pm}+\ldots
\end{array}
$$

3. Collecting the same terms of $e$ in the PDE inside and outside the sheet, and the conditions for the Dirichlet and normal traces on $\Gamma_{ \pm}^{e}$.

4. In order to simplify the problem, we introduce a problem satisfied by an approximation $E_{e}^{k}$ of the expression $E_{0}(x)+e E_{1}(x)+e^{2} E_{2}(x)+\ldots+e^{k} E_{k}(x)$ up to a residual term in $\mathscr{O}\left(e^{k+1}\right)$.

\subsubsection{Equivalent Model of Order 1}

The first order approximate solution $E_{0}$ satisfies the perfect electric conductor (PEC) boundary conditions on $\Gamma$. In this case, the two domains $\Omega_{-}$and $\Omega_{+}$are perfectly isolated. $E_{0}$ solves

$$
\left\{\begin{array}{lll}
\operatorname{curlcurl} E_{0}^{-} & =0 & \text { in } \Omega_{-}, \\
n \times\left(E_{0}^{-} \times n\right) & =0 & \text { on } \Gamma, \\
\operatorname{curlcurl} E_{0}^{+} & =i \omega \mu_{+} J_{0} & \text { in } \Omega_{+}, \\
n \times\left(E_{0}^{+} \times n\right) & =0 & \text { on } \Gamma, \\
\operatorname{div} E_{0}^{ \pm} & =0 & \text { in } \Omega_{ \pm}, \\
\int_{\Gamma_{ \pm}} E_{0}^{ \pm} \cdot n d S & =0 & \text { on } \Gamma_{ \pm}, \\
E_{0} & =O\left(\frac{1}{|x|}\right) & \text { as }|x| \rightarrow \infty .
\end{array}\right.
$$




\subsubsection{Equivalent Model of Order 2}

Let $u$ be a vector field on $\Gamma$, we denote by

$$
\gamma_{D} u=n \times(u \times n), \gamma_{N} u=\operatorname{curl} u \times n, \text { and } \gamma_{n} u=u \cdot n,
$$

here $n$ is the unit normal vector on $\Gamma$ which is oriented from the inner domain towards the outer domain. The term $E_{1}$ satisfies the following problem:

$$
\left\{\begin{array}{lll}
\operatorname{curlcurl} E_{1} & =0 & \text { in } \Omega_{ \pm}, \\
\operatorname{div} E_{1} & =0 & \text { in } \Omega_{ \pm}, \\
E_{1} & =O\left(\frac{1}{|x|}\right) & \text { as }|x| \rightarrow \infty, \\
\left(\begin{array}{l}
{\left[\gamma_{D} E_{1}\right]_{\Gamma}} \\
\left\{\gamma_{D} E_{1}\right\}_{\Gamma}
\end{array}\right) & =-\left(\begin{array}{ll}
C_{1} & C_{3} \\
C_{3} & C_{2}
\end{array}\right)\left(\begin{array}{c}
\left\{\frac{1}{\mu}\left(\gamma_{N} E_{0}\right)\right\}_{\Gamma} \\
{\left[\frac{1}{\mu}\left(\gamma_{N} E_{0}\right)\right]_{\Gamma}}
\end{array}\right) & \text { on } \Gamma,
\end{array}\right.
$$

where (Victor Péron, 2017)

$$
\begin{aligned}
& C_{1}=\{\mu\}-2 \frac{\mu_{0}^{c}}{\gamma} \tanh \left(\frac{\gamma}{2}\right) \quad, \quad C_{2}=\frac{\{\mu\}}{4}-\frac{\mu_{0}^{c}}{2 \gamma} \operatorname{coth}\left(\frac{\gamma}{2}\right), \\
& C_{3}=\frac{1}{4}[\mu] .
\end{aligned}
$$

Adding (2.14) multiplied by $e$ to the conditions for $E_{0}$ and by replacing $E_{0}+e E_{1}$ on the left side by the $E_{e}^{1}$ and by replacing $e E_{0}$ on the right hand side by $e E_{1}^{e}$. We obtain the second order approximate solution $E_{e}^{1}$ that solves the system

$$
\left\{\begin{array}{lll}
\operatorname{curlcurl} E_{e}^{1} & =i \omega \mu J_{0} & \text { in } \Omega_{ \pm}, \\
\operatorname{div} E_{e}^{1} & =0 & \text { in } \Omega_{ \pm}, \\
E_{e}^{1} & =O\left(\frac{1}{|x|}\right) & \text { as }|x| \rightarrow \infty \\
\left(\begin{array}{ll}
{\left[\gamma_{D} E_{e}^{1}\right]_{\Gamma}} \\
\left\{\gamma_{D} E_{e}^{1}\right\}_{\Gamma}
\end{array}\right) & =-e\left(\begin{array}{ll}
C_{1} & C_{3} \\
C_{3} & C_{2}
\end{array}\right)\left(\begin{array}{c}
\left\{\frac{1}{\mu}\left(\gamma_{N} E_{e}^{1}\right)\right\}_{\Gamma} \\
{\left[\frac{1}{\mu}\left(\gamma_{N} E_{e}^{1}\right)\right]_{\Gamma}}
\end{array}\right) & \text { on } \Gamma,
\end{array}\right.
$$

Symmetric case with permeability $\mu_{0}^{c}=\mu_{+}=\mu_{-}=\mu_{0}$ leads to

$$
\left\{\begin{array}{lll}
\operatorname{curlcurl} E_{e}^{1} & =i \omega \mu J_{0} & \text { in } \Omega_{ \pm}, \\
\operatorname{div} E_{e}^{1} & =0 & \text { in } \Omega_{ \pm}, \\
E_{e}^{1} & =O\left(\frac{1}{|x|}\right) & \text { as }|x| \rightarrow \infty, \\
\left(\begin{array}{l}
{\left[\gamma_{D} E_{e}^{1}\right]_{\Gamma}} \\
\left\{\gamma_{D} E_{e}^{1}\right\}_{\Gamma}
\end{array}\right) & =-e\left(\begin{array}{ll}
K_{1} & 0 \\
0 & K_{2}
\end{array}\right)\left(\begin{array}{c}
\left\{\left(\gamma_{N} E_{e}^{1}\right)\right\}_{\Gamma} \\
{\left[\left(\gamma_{N} E_{e}^{1}\right)\right]_{\Gamma}}
\end{array}\right) & \text { on } \Gamma,
\end{array}\right.
$$

where

$$
K_{1}=1-2 \frac{1}{\gamma} \tanh \left(\frac{\gamma}{2}\right) \quad, \quad K_{2}=\frac{1}{4}-\frac{1}{2 \gamma} \operatorname{coth}\left(\frac{\gamma}{2}\right)
$$

\section{Boundary Element Method}

As out of the layer we mainly consider a non-conductive linear homogeneous domain and an open boundary problem, we can avoid the volume mesh using BEM that requires only $2 \mathrm{D}$ elements on the surfaces. After introducing the functional spaces, the potentials, and the general representation formula in sections 3.1, 3.2 and 3.3 respectively, we formulate the integral equations, the variational formulations, and the Galerkin discretisation using special basis functions of the terms of expansion $E_{0}$ in section $3.4, E_{1}$ in section 3.5 , and the equivalent model of second order $E_{e}^{1}$ in section 3.6. Note that we consider $\mu_{-}=\mu_{+}=\mu_{0}$ all over this section. 


\subsection{Functional Spaces}

The spaces that are related to the traces of vector fields in $H\left(\operatorname{curl}, \Omega_{ \pm}\right)$onto $\Gamma$ must be considered using Boundary Integral Equations. We will use the following spaces of tangential vector fields on $\Gamma$, defined in (A. Buffa and M. Fortin, 1999),

- $H_{\|}^{\frac{1}{2}}(\Gamma)$ represents the tangential surface vector fields that are in $H^{\frac{1}{2}}\left(\Gamma_{i}\right)$ for each smooth component $\Gamma_{i}$ of $\Gamma$, and provides the weak tangential continuity across the edges of $\Gamma_{i}$.

- $H_{\perp}^{\frac{1}{2}}(\Gamma)$ provides the weak normal continuity.

For a smooth boundary $\Gamma$, these spaces coincide with that of tangential surface vector fields in $H^{\frac{1}{2}}(\Gamma)$. We denote by $H_{\|}^{-\frac{1}{2}}(\Gamma)$, and $H_{\perp}^{-\frac{1}{2}}(\Gamma)$ the dual spaces of $H_{\|}^{\frac{1}{2}}(\Gamma)$ and $H_{\perp}^{\frac{1}{2}}(\Gamma)$, respectively.

We denote by $\operatorname{curl}_{\Gamma}$ the tangential rotational operator and by $\operatorname{curl}_{\Gamma}$ the surface rotational operator:

$$
\begin{gathered}
\forall \phi \in C^{\infty}(\Gamma), \quad \operatorname{curl}_{\Gamma} \phi=\left(\nabla_{\Gamma} \phi\right) \times n, \\
\forall v \in\left(C^{\infty}(\Gamma)\right)^{3}, \quad \operatorname{curl}_{\Gamma} v=\operatorname{div}_{\Gamma}(v \times n),
\end{gathered}
$$

where $\nabla_{\Gamma}$ and $\operatorname{div}_{\Gamma}$ are respectively the tangential gradient and the surface divergence on $\Gamma$.

These surface differential operators are used to define the spaces $H_{\perp}^{-\frac{1}{2}}\left(\operatorname{curl}_{\Gamma}, \Gamma\right)$, and $H_{\|}^{-\frac{1}{2}}\left(\operatorname{div}_{\Gamma}, \Gamma\right)$ introduced in (A. Buffa and M. Fortin, 1999) by

$$
\begin{aligned}
& H_{\perp}^{-\frac{1}{2}}\left(\operatorname{curl}_{\Gamma}, \Gamma\right)=\left\{v \in H_{\perp}^{-\frac{1}{2}}(\Gamma), \operatorname{curl}_{\Gamma} v \in H^{-\frac{1}{2}}(\Gamma)\right\}, \\
& H_{\|}^{-\frac{1}{2}}\left(\operatorname{div}_{\Gamma}, \Gamma\right)=\left\{w \in H_{\|}^{-\frac{1}{2}}(\Gamma), \operatorname{div}_{\Gamma} w \in H^{-\frac{1}{2}}(\Gamma)\right\} .
\end{aligned}
$$

Another property in (A. Buffa and M. Fortin, 1999, sec. 4) is that $H_{\perp}^{-\frac{1}{2}}\left(\operatorname{curl}_{\Gamma}, \Gamma\right)$ and $H_{\|}^{-\frac{1}{2}}\left(\operatorname{div}_{\Gamma}, \Gamma\right)$ are dual of each others, when the space of $L^{2}$-integrable vector fields $L^{2}(\Gamma)$ is used as pivot space. According to (R. Hiptmair, 2002, sec. 3), $H_{\perp}^{-\frac{1}{2}}\left(\operatorname{curl}_{\Gamma}, \Gamma\right), H_{\|}^{-\frac{1}{2}}\left(\operatorname{div}_{\Gamma} 0, \Gamma\right)$ and $H^{\frac{1}{2}}(\Gamma)$ are the suitable spaces for the Dirichlet data $\gamma_{D}$, the Neumann data $\gamma_{N}$, and the normal data $\gamma_{n}$. respectively, where $H_{\|}^{-\frac{1}{2}}\left(\operatorname{div}_{\Gamma} 0, \Gamma\right)=$ $\operatorname{Ker}\left(\operatorname{div}_{\Gamma}, H_{\|}^{-\frac{1}{2}}\left(\operatorname{div}_{\Gamma}, \Gamma\right)\right)$.

\subsection{Potentials}

We recall the definition of the green function for laplace operator (kernel)

$$
G(x, y)=\frac{1}{4 \pi} \frac{1}{|x-y|}, x, y \in \mathbb{R}^{3}, x \neq y .
$$

The scalar single-layer potential is given by

$$
\Psi_{V}(\Phi)(x)=\int_{\Gamma} \Phi(y) G(x, y) d S(y), \quad x \notin \Gamma .
$$


For any tangential vector field $\lambda$ on $\Gamma$ we define the vectorial single-layer potential by

$$
\Psi_{A}(\lambda)(x)=\int_{\Gamma} \lambda(y) G(x, y) d S(y), x \notin \Gamma,
$$

the vectorial Newton potential

$$
N(\lambda)(x)=\int_{\mathbb{R}^{3}} \lambda(y) G(x, y) d y,
$$

and the vectorial double-layer potential

$$
\Psi_{M}(u)=\operatorname{curl}_{A}(R u), \quad R u=n \times u .
$$

\subsection{Boundary Integral Equations}

Theorem 3.1 (R. Hiptmair, 2002; R. Hiptmair and J. Ostrowski, 2005) Let $E \in L^{2}\left(\mathbb{R}^{3}\right)$ with curlE $\in$ $L^{2}\left(\Omega_{ \pm}\right)$. If a vector field $E: \Omega_{ \pm} \longrightarrow \mathbb{C}^{3}$ satisfies

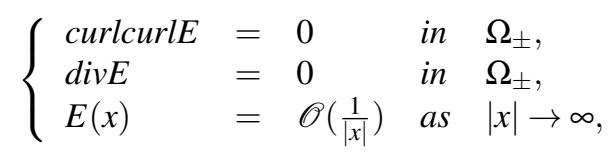

then it satisfies the following transmission formula

$$
E=\Psi_{M}\left(\left[\gamma_{D} E\right]_{\Gamma}\right)+\Psi_{A}\left(\left[\gamma_{N} E\right]_{\Gamma}\right)-\operatorname{grad} \Psi_{V}\left(\left[\gamma_{n} E\right]_{\Gamma}\right) .
$$

Applying the trace operators $\gamma_{D}$. to the representation formula leads to a boundary-integral equation. For this reason we define the following operators,

$$
\begin{aligned}
& K=\left\{\gamma_{D} \Psi_{M}\right\}_{\Gamma} \quad, \quad V=\left\{\gamma_{D} \Psi_{A}\right\}_{\Gamma} \\
& Q=\left\{\gamma_{D} \Psi_{V}\right\}_{\Gamma}
\end{aligned}
$$

\subsection{Equivalent Model of Order 1}

Recall that $E_{0}$ satisfies (2.13).

\subsubsection{Integral Equations for $E_{0}$}

In order to write the integral equation in the case where we have an excitation by $J_{0}$, we introduce the Newton potential representing the source term

$$
E_{s}(x)=i \omega \mu \int_{\mathbb{R}^{3}} J_{0}(y) G(x, y) d y,
$$

It is sufficient to consider the representation formula of $E_{0}$ in $\Omega_{+}$, using Theorem 3.1 we state the representation formula as

$$
E_{0}=-\Psi_{M}\left(\gamma_{D}^{+} E_{0}\right)-\Psi_{A}\left(\gamma_{N}^{+} E_{0}\right)-\operatorname{grad} \Psi_{v}\left(\gamma_{n}^{+} E_{0}\right)+E_{s} .
$$

Applying $\gamma_{D}^{+}$to the representation formula, we find for $E_{0}$

$$
\gamma_{D}^{+} E_{0}=\left(\frac{1}{2} \mathbb{I}-K\right)\left(\gamma_{D}^{+} E_{0}\right)-V\left(\gamma_{N}^{+} E_{0}\right)-\operatorname{grad} Q\left(\gamma_{n} E_{0}\right)+\gamma_{D}^{+} E_{S},
$$

this equation is set in $H_{\perp}^{-\frac{1}{2}}\left(\operatorname{curl}_{\Gamma}, \Gamma\right)$ which is the appropriate space of Dirichlet data. 


\subsubsection{Variational Formulation for $E_{0}$}

We obtain an equivalent variational formulation by testing against function from the dual space of $H_{\perp}^{-\frac{1}{2}}\left(\operatorname{curl}_{\Gamma}, \Gamma\right)$.

The dual space of $H_{\perp}^{-\frac{1}{2}}\left(\operatorname{curl}_{\Gamma}, \Gamma\right)$ being the space $H_{\|}^{-\frac{1}{2}}\left(\operatorname{div}_{\Gamma}, \Gamma\right)$, we have to solve

Find $\gamma_{N}^{+} E_{0} \in H_{\|}^{-\frac{1}{2}}\left(\operatorname{div}_{\Gamma}, \Gamma\right)$, such that

$$
<V\left(\gamma_{N}^{+} E_{0}\right), B_{1}>_{\Gamma}=<\gamma_{D}^{+} E_{S}, B_{1}>_{\Gamma},
$$

for every $B_{1} \in H_{\|}^{-\frac{1}{2}}\left(\operatorname{div}_{\Gamma} 0, \Gamma\right)$.

Since

$$
\begin{gathered}
<\operatorname{grad} Q(\Phi), \lambda>_{\Gamma}=0 \quad \text { for every } \lambda \in H_{\|}^{-\frac{1}{2}}\left(\operatorname{div}_{\Gamma} 0, \Gamma\right),(\mathrm{R} . \text { Hiptmair, 2002) } \\
\gamma_{D}^{+} E_{0}=0,
\end{gathered}
$$

where $<f, g>_{\Gamma}=\int_{\Gamma} f(x) g(x) d \Gamma(x)$.

\subsubsection{Galerkin Discretization}

The Neumann data $\lambda=\gamma_{N}^{+} E_{0}$ requires an approximation by means of solenoidal $\operatorname{div}_{\Gamma^{-}}$-conforming surface elements. They are supplied by the space of divergence-free lowest order Raviart- Thomas elements $\mathbf{R T}(\Gamma)$ on $\Gamma$ (P. A. Raviart and J. M. Thomas, 1977). If $\Gamma$ is simply connected, then $\mathbf{R T}(\Gamma)=\operatorname{curl}_{\Gamma} N_{1}(\Gamma)$, where $N_{1}(\Gamma)$ is the space of nodal functions of degree 1 .

$\lambda$ is approximated by the discrete field :

$$
\lambda_{h}=\sum_{i=1}^{N} \lambda^{i} W_{c u r l N}^{i}
$$

where $N$ is the number of nodes, the coefficients $\lambda^{i}$, s are the values of $\lambda_{h}$ at node $i$, and $W_{\text {curlN }}^{i}$ is the surface rotational operator of nodal shape function of degree 1 corresponds to the node $i$.

Applying Galerkin Method, the test functions $B_{1}$ should be replaced by $W_{\text {curlN }}^{j}$. We can now state the discretised formulation as:

Find $\lambda^{i} \in \mathbb{R}^{n}$, such that

$$
\sum_{i=1}^{N} \lambda^{i}<V\left(W_{\text {curlN }}^{i}\right), W_{\text {curl }}^{j}>_{\Gamma}=<\gamma_{D}^{+} E_{s}, W_{\text {curlN }}^{j}>_{\Gamma},
$$

for $j=1 . . N$. 


\subsection{Strong Formulation for $E_{1}$}

Recall that $E_{1}$ satisfies in the symmetric case (see section 2.2.3)

$$
\left\{\begin{array}{lll}
\operatorname{curlcurl} E_{1} & =0 & \text { in } \Omega_{ \pm}, \\
\operatorname{div} E_{1} & =0 & \text { in } \Omega_{ \pm}, \\
E_{1} & =O\left(\frac{1}{|x|}\right) & \text { as }|x| \rightarrow \infty, \\
\left(\begin{array}{l}
{\left[\gamma_{D} E_{1}\right]_{\Gamma}} \\
\left\{\gamma_{D} E_{1}\right\}_{\Gamma}
\end{array}\right) & =\left(\begin{array}{ll}
K_{1} & 0 \\
0 & K_{2}
\end{array}\right)\left(\begin{array}{c}
\left\{\left(\gamma_{N} E_{0}\right)\right\}_{\Gamma} \\
{\left[\left(\gamma_{N} E_{0}\right)\right]_{\Gamma}}
\end{array}\right) & \text { on } \Gamma,
\end{array}\right.
$$

where

$$
K_{1}=-1+2 \frac{1}{\gamma} \tanh \left(\frac{\gamma}{2}\right) \quad, \quad K_{2}=-\frac{1}{4}+\frac{1}{2 \gamma} \operatorname{coth}\left(\frac{\gamma}{2}\right)
$$

\subsubsection{Variational Formulation for $E_{1}$}

We write the representation formula of $E_{1}$ in $\Omega_{+}$as

$$
E_{1}=-\Psi_{M}\left(\gamma_{D}^{+} E_{1}\right)-\Psi_{A}\left(\gamma_{N}^{+} E_{1}\right)-\operatorname{grad} \Psi_{v}\left(\gamma_{n}^{+} E_{1}\right)
$$

Applying the Dirichlet trace, we arrive at

$$
\gamma_{D}^{+} E_{1}=\left(\frac{1}{2} \mathbb{I}-K\right)\left(\gamma_{D}^{+} E_{1}\right)-V\left(\gamma_{N}^{+} E_{1}\right)-\operatorname{grad} Q\left(\gamma_{n} E_{1}\right) .
$$

By the transmission conditions of the $E_{1}$ model (3.10), we can write:

$$
\gamma_{D}^{+} E_{1}=\frac{K_{1}}{4} \gamma_{N}^{+} E_{0}+K_{2} \gamma_{N}^{+} E_{0}
$$

Substitute (3.13) in (3.12) we obtain the variational formulation

Find $\gamma_{N}^{+} E_{1} \in H_{\|}^{-\frac{1}{2}}\left(\operatorname{div}_{\Gamma}, \Gamma\right)$, such that

$$
<V\left(\gamma_{N}^{+} E_{1}\right), B_{1}>_{\Gamma}=<\left(-\frac{1}{2} \mathbb{I}-K\right)\left(\frac{K_{1}}{4} \gamma_{N}^{+} E_{0}+K_{2} \gamma_{N}^{+} E_{0}\right), B_{1}>_{\Gamma}
$$

for every $B_{1} \in H_{\|}^{-\frac{1}{2}}\left(\operatorname{div}_{\Gamma} 0, \Gamma\right)$.

\subsubsection{Galerkin Discretization}

Let $\alpha=\gamma_{N}^{+} E_{1}, \alpha$ is discretized by $\alpha_{h}=\sum_{i=1}^{N} \alpha^{i} W_{c u r l N}^{i}$ where $N$ is the number of nodes, the coefficients $\alpha^{i}$,s are the values of $\alpha_{h}$ at node $i$. Applying Galerkin Method, we can state the discretised formulation as:

Find $\alpha^{i} \in \mathbb{R}^{n}$, such that

$$
\sum_{i=1}^{N} \alpha^{i}<V\left(W_{\text {curl }}^{i}\right), W_{\text {curlN }}^{j}>_{\Gamma}=<\left(-\frac{1}{2} \mathbb{I}-K\right)\left(\frac{K_{1}}{4} \gamma_{N}^{+} E_{0}+K_{2} \gamma_{N}^{+} E_{0}\right), W_{\text {curlN }}^{j}>_{\Gamma}
$$

for $j=1 . . N$. 


\subsection{Equivalent Model of Order 2}

Recall that $E_{e}^{1}$ satisfies (2.16).

\subsubsection{Variational Formulation for $E_{e}^{1}$}

For $E_{e}^{1} \in L^{2}\left(\mathbb{R}^{3}\right)$, the representation formulas can be given by

$$
\begin{gathered}
E_{e}^{1}=-\Psi_{M}\left(\gamma_{D}^{+} E_{e}^{1}\right)-\Psi_{A}\left(\gamma_{N}^{+} E_{e}^{1}\right)-\operatorname{grad} \Psi_{V}\left(\gamma_{n}^{+} E_{e}^{1}\right)+E_{s} \text { in } \Omega_{+}, \\
E_{e}^{1}=\Psi_{M}\left(\gamma_{D}^{-} E_{e}^{1}\right)+\Psi_{A}\left(\gamma_{N}^{-} E_{e}^{1}\right)+\operatorname{grad} \Psi_{V}\left(\gamma_{n}^{-} E_{e}^{1}\right) \text { in } \Omega_{-} .
\end{gathered}
$$

Applying the Dirichlet trace on (3.16) and (3.17), we obtain the following integral equations

$$
\begin{gathered}
V\left(\gamma_{N}^{+} E_{e}^{1}\right)+\left(\frac{1}{2} \mathbb{I}+K\right)\left(\gamma_{D}^{+} E_{e}^{1}\right)=\gamma_{D}^{+} E_{s}-\operatorname{grad}_{\Gamma} Q\left(\gamma_{n}^{+} E_{e}^{1}\right) \\
-V\left(\gamma_{N}^{-} E_{e}^{1}\right)+\left(\frac{1}{2} \mathbb{I}-K\right)\left(\gamma_{D}^{-} E_{e}^{1}\right)=\operatorname{grad}_{\Gamma} Q\left(\gamma_{n}^{+} E_{e}^{1}\right) .
\end{gathered}
$$

Using the transmission conditions, we obtain the following equalities

$$
\begin{gathered}
\gamma_{D}^{+} E_{e}^{1}=D_{0} \gamma_{N}^{+} E_{e}^{1}+D_{1} \gamma_{N}^{-} E_{e}^{1}, \\
\gamma_{D}^{-} E_{e}^{1}=-D_{1} \gamma_{N}^{+} E_{e}^{1}-D_{0} \gamma_{N}^{-} E_{e}^{1}
\end{gathered}
$$

where $D_{0}=e\left(\frac{K_{1}}{4}+K_{2}\right)$, and $D_{1}=e\left(\frac{K_{1}}{4}-K_{2}\right)$.

Substitute the transmission conditions (3.20) and (3.21) in the integral equations (3.18) and (3.19), we find the variational formulation

Find $\gamma_{N}^{+} E_{e}^{1}, \gamma_{N}^{-} E_{e}^{1} \in H_{\|}^{-\frac{1}{2}}\left(\operatorname{div}_{\Gamma}, \Gamma\right)$, such that

$$
\begin{array}{r}
<\left(V+\frac{1}{2} D_{0} \mathbb{I}+D_{0} K\right)\left(\gamma_{N}^{+} E_{e}^{1}\right), B_{1}>_{\Gamma}+<\left(\frac{1}{2} D_{1} \mathbb{I}+D_{1} K\right)\left(\gamma_{N}^{-} E_{e}^{1}\right), B_{1}>_{\Gamma}=<\gamma_{D}^{+} E_{s}, B_{1}>_{\Gamma} \\
<\left(-\frac{1}{2} D_{1} \mathbb{I}+D_{1} K\right)\left(\gamma_{N}^{+} E_{e}^{1}\right), B_{2}>_{\Gamma}+<\left(-V-\frac{1}{2} D_{0} \mathbb{I}+D_{0} K\right)\left(\gamma_{N}^{-} E_{e}^{1}\right), B_{2}>_{\Gamma}=0
\end{array}
$$

for every $B_{1}, B_{2} \in H_{\|}^{-\frac{1}{2}}\left(\operatorname{div}_{\Gamma} 0, \Gamma\right)$.

\subsubsection{Galerkin Discretization}

Let $\beta=\gamma_{N}^{+} E_{e}^{1}$ and $\beta^{\prime}=\gamma_{N}^{-} E_{e}^{1} . \beta$ and $\beta^{\prime}$ are approximated as $\beta_{h}=\sum_{i=1}^{N} \beta^{i} W_{c u r l N}^{i}$ and $\beta_{h}^{\prime}=\sum_{i=1}^{N} \beta^{\prime i} W_{c u r l N}^{i}$ respectively, the coefficients $\beta^{i}$, s and $\beta^{\prime i}$ 's are the values of $\beta_{h}$ and $\beta_{h}^{\prime}$ respectively at node $i$. Applying Galerkin Method, we can state the discretised formulation as: 
Find $\beta^{i}, \beta^{i} \in \mathbb{R}^{n}$, such that

$\sum_{i=1}^{N} \beta^{i}<\left(V+\frac{1}{2} D_{0} \mathbb{I}+D_{0} K\right)\left(W_{\text {curl } N}^{i}\right), W_{\text {curl } N}^{j}>_{\Gamma}+\sum_{i=1}^{N} \beta^{i}<\left(\frac{1}{2} D_{1} \mathbb{I}+D_{1} K\right)\left(W_{\text {curlN }}^{i}\right), W_{\text {curlN }}^{j}>_{\Gamma}=<\gamma_{D}^{+} E_{S}, W_{\text {curlN }}^{j}>_{\Gamma}$,

$$
\sum_{i=1}^{N} \beta^{i}<\left(-\frac{1}{2} D_{1} \mathbb{I}+D_{1} K\right)\left(W_{\text {curl } N}^{i}\right), W_{\text {curl } N}^{j}>_{\Gamma}+\sum_{i=1}^{N} \beta^{i i}<\left(-V-\frac{1}{2} D_{0} \mathbb{I}+D_{0} K\right)\left(W_{c u r l N}^{i}\right), W_{\text {curl } N}^{j}>_{\Gamma}=0
$$

for $j=1 . . N$.

\subsection{Implementation}

We implement our model in the platform «MIPSE » of the G2Elab. For actual implementation, we need integral representations for the boundary integral operators. In particular, we explicit the following results.

Proposition 3.1 (R. Hiptmair and J. Ostrowski, 2005) For $\lambda \in L^{\infty}(\Gamma)$

- $V(\lambda)=\left\{\gamma_{D}\right\} \circ \Psi_{A}(\lambda)=\int_{\Gamma} \lambda(y) G(x, y) d S(y)$

- $\tilde{K}(\lambda)=\left\{\gamma_{N}\right\} \circ \Psi_{A}(\lambda)=\int_{\Gamma}\left(\frac{\partial G(x, y)}{\partial n(x)} \lambda(y)-\operatorname{grad}_{x} G(x, y)(\lambda(y) \cdot n(x))\right) d S(y)$

Theorem 3.2 (R. Hiptmair and J. Ostrowski, 2005) If $\operatorname{Re}\left(k^{2}\right) \geq 0$, the boundary operators $\tilde{K}$ and $K$ satisfy

$$
<\tilde{K} \mu, v>_{\Gamma}=-<\mu, K v>_{\Gamma}
$$

for every $\mu \in H_{\|}^{-\frac{1}{2}}\left(\operatorname{div}_{\Gamma} 0, \Gamma\right)$ and $v \in H_{\perp}^{-\frac{1}{2}}\left(\operatorname{curl}_{\Gamma}, \Gamma\right)$

\section{Numerical Results}

In this section we validate the accuracy of the integral equations, and we provide three examples to validate our model. Examples 1 and 2 satisfy the theoretical condition where closed curved thin layer is considered. However, "Example 3" is provided to show the effectiveness of our model even in open domains that does not satisfy the theoretical condition.

\subsection{Validation of Integral Equations}

To verify the efficiency of the integral equations, we consider a sphere of radius $r=1 \mathrm{~m}$ that satisfies the conditions of (PEC) which is the case for the model of order 1 . The frequency $f=100 \mathrm{kHz}$, the source field is excited by a uniform magnetic field in $z$-direction. We compare the numerical solution calculated on an arc of a circle of radius $1.3 \mathrm{~m}$ to an analytical solution, we obtain an error $\left\|H_{\text {analytic }}-H_{0}\right\|_{2}=2.6 \times 10^{-6}$. It validates the accuracy of the integral equations. 


\subsection{Example 1}

We consider a sphere with radius $0.99 \mathrm{~m}$, surrounded by a conductive sheet of thickness $e=2 \mathrm{~cm}$ with $\tilde{\sigma}=10^{+3}$ and $f=1 \mathrm{kHz}$. The skin depth $\delta=0.0010 \mathrm{~m}$ and the source is excited by a uniform magnetic field $H_{0}^{s}=1 \vec{z}$.

We calculate the external magnetic field on an arc of circle at radius $1.3 \mathrm{~m}$ using a mesh of 384 elements. In
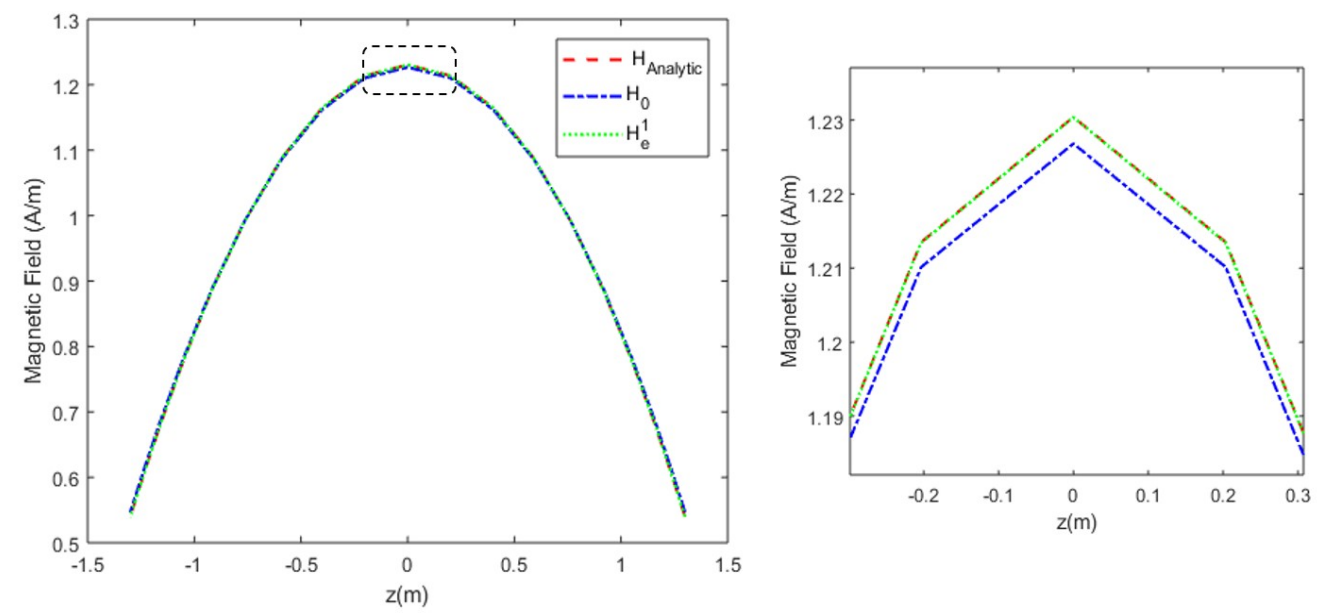

Figure 2: The magnetic fields $H_{0}$ and $H_{e}^{1}$ on the arc of radius $1.3 \mathrm{~m}$ compared to the analytical solution

figure 2, we plot on the arc the magnetic field calculated by the model of order 1, and the model of order 2 . We compare the results to the analytical solution to obtain the following errors

$$
\begin{gathered}
\left\|H_{\text {analytic }}-H_{0}\right\|_{2}=0.0196, \\
\left\|H_{\text {analytic }}-H_{e}^{1}\right\|_{2}=7 \times 10^{-4},
\end{gathered}
$$

which shows a good agreement, and confirms the theoretical approach.

\subsection{Example 2}

The aim of this example is to show the robustness of the equivalent models versus the parameter $\tilde{\sigma}$. we consider a sphere of radius $r=0.98 \mathrm{~m}$ surrounded by a conductive sheet of thickness $e=4 \mathrm{~cm}$, the skin depth is a function of $\tilde{\sigma}$. In figure 3 we show the relative $L^{2}$-errors of the solutions $H_{0}$ and $H_{e}^{1}$ of the equivalent models of order 1 and 2 versus the parameter $\tilde{\sigma}$ for $f=10 \mathrm{kHz}$, where the source field is excited by a uniform magnetic field $H_{0}^{s}=1 \vec{z}$. Similarly the error is calculated on an arc of circle at radius $1.3 \mathrm{~m}$.

The equivalent model of order 1 shows a good agreement, we observe a small error in a wide range of skin depths, the interval where the skin depths is small compared to $e$ or of the same order, this result corresponds to the theoretical assumption. Adding the term $e H_{1}$ improves the result only in the interval of high conductivity i.e. where $\delta \leq e$, that is because $H_{1}$ depends on $H_{0}$ (see 2.14). The equivalent model of order 2 gives very small errors for all ranges of the skin depth, from very small to very large. This result demonstrates the effectiveness of the model of order 2. 


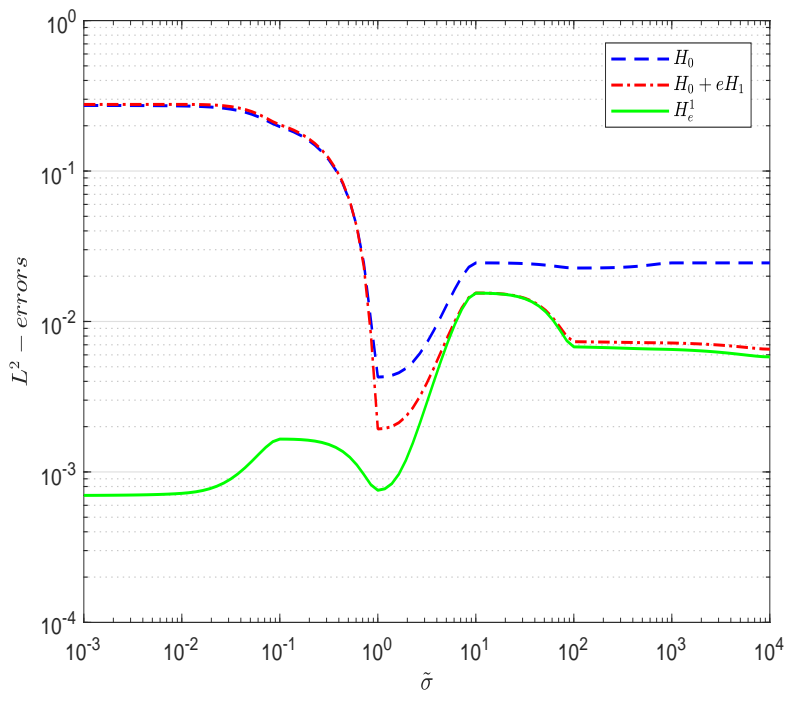

Figure 3: Relative $L^{2}$ errors of the solutions $H_{0}$ and $H_{e}^{1}$ of the equivalent models of order 1 and 2 versus the parameter $\tilde{\sigma}$ for $e=4 \mathrm{~cm}$

\subsection{Example 3}

In this example we provide an open boundary problem, where a cylindrical sheet of radius $=1 \mathrm{~m}$, height $=3 \mathrm{~m}$, thickness $e=2 \mathrm{~cm}$, and frequency $f=10 \mathrm{kHz}$ is considered. The source is excited by a spire of radius $R=1.1 \mathrm{~m}$ and a current of $10 \mathrm{~A}$, note that both the cylinder and the spire have the same central axis, and their center is the origin of the coordinate system. We compute the norm of the magnetic field on a segment that connect the two points $(1.3,0,-2)$ and $(1.3,0,+2)$ and we compare the results to the $2 \mathrm{~d}$ axisymmetric formulation in Comsol that discretized using the Finite Element Method. The corresponding errors are presented in Table 1, we observe that the model of order 2 still works well, that improves the effectiveness of the second order model even in open domains. The errors obtained are strongly lower than the amplitude of the components of the reduced magnetic field $H_{e \text { Reduced }}^{1}$ (reaction field), see Fig. 4 for the $x$-component and see Fig. 5 for the $z$-component. This seems to indicate that, in this configuration, no particular care have to be taken even if the surface is not simply connected.

Comparing the time consumption and the number of elements of the mesh used by each method (see Table 1), we deduce that the boundary element method reduces the time consumption as it needs a reduced number of elements for discretisation.

Table 1: The $L^{\infty}$-errors, the time consumption and the number of elements of the mesh used in simulations done by Comsol, the model of order 1, and the model of order 2.

\begin{tabular}{cccc}
\hline & Nb of elements & Time & $\|\cdot\|_{\infty}$ \\
\hline Comsol (Reference) & 1771542 & $132 \mathrm{~s}$ & \\
Model Order 1 & 544 & $25 \mathrm{~s}$ & 0.128 \\
Model Order 2 & 544 & $56 \mathrm{~s}$ & 0.021 \\
\hline
\end{tabular}




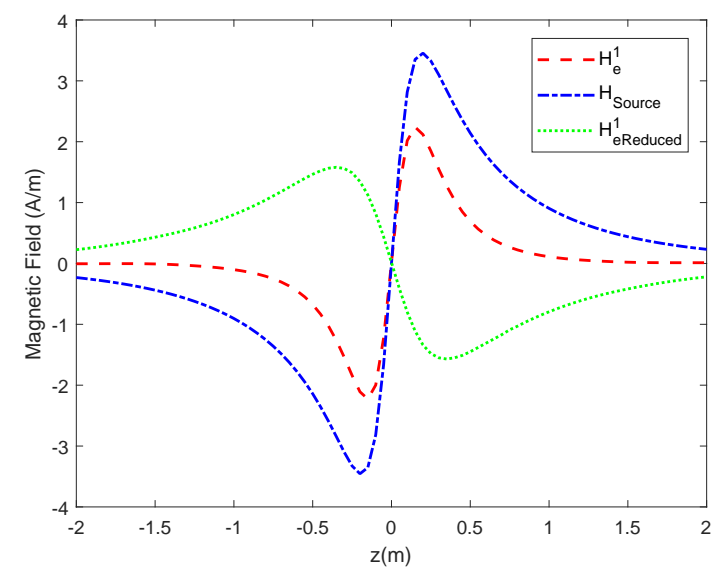

Figure 4: The $x$-component of the real part of the total magnetic fields $H_{e}^{1}$, the reduced magnetic fields $H_{e \text { Reduced }}^{1}$ and the source field $H_{\text {Source }}$.

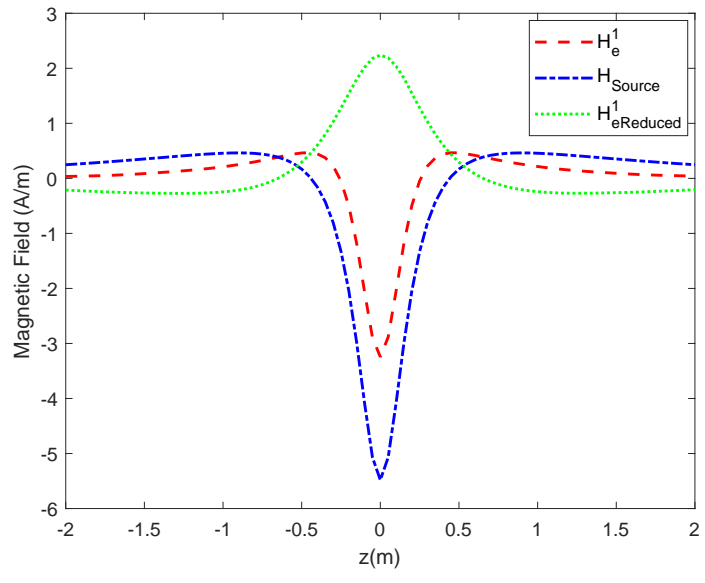

Figure 5: The $z$-component of the real part of the total magnetic fields $H_{e}^{1}$, the reduced magnetic fields $H_{e \text { Reduced }}^{1}$ and the source field $H_{\text {Source }}$.

\section{Conclusion}

A second order equivalent model for eddy current problems with a thin layer in $3 D$ is proposed and discretized using the Boundary Element Method. The model is validated and shows a good agreement. The high order model provides more precision than the classical model, it is robust and can be used from low to very high conductivity. The discretization method shows a great success in the precision of results and in reducing the number of unknowns and the time consumption.

\section{Acknowledgements}

We acknowledge the collaboration of our colleagues B. BANNWARTH, and G. MEUNIER from G2Elab who participated in the implementation of the model. A short version of this paper was presented at 9th European Conference on Numerical Methods in Electromagnetism, Paris, in November 2017.

\section{References}

Victor Péron, Kersten Schmidt, Marc Duruflé (2016), Equivalent transmission conditions for the timeharmonic Maxwell equations in 3D for a medium with a highly conductive thin sheet, SIAM Journal on Applied Mathematics, Society for Industrial and Applied Mathematics, 76 (3), pp.1031-1052. $<10.1137 / 15$ M1012116>. <hal-01260111v2>. 
R. Hiptmair (2002), Symmetric coupling for eddy current problems, SIAM J. Numer. Anal. 40,pp. 41-65.

A. Buffa and M. Fortin (1999), On traces for functional spaces related to Maxwell's equations.Part I: An integration by parts formula in Lipschitz polyhedra, Tech. Rep. 1147, Instituto di Analisi Numerica, CNR, Pavia, Italy.

A. Buffa and M. Fortin (1999), On traces for functional spaces related to Maxwell's equations.Part II: Hodge decompositions on the boundary of Lipschitz polyhedra and applicatons, Tech. Rep. 1147, Instituto di Analisi Numerica, CNR, Pavia, Italy.

P. A. Raviart and J. M. Thomas (1977), A mixed Finite Element Method for second order elliptic problems, vol. 606 of Springer lecture notes in Mathematics, Springer-Vergal, New York,pp. 292315.

Victor Péron (2017), Impedance transmission conditions for eddy current problems, https://hal.inria.fr/hal01505612 .

M. Issa, B. Bannwarth, O. Chadebec, G. Meunier, J-R. Poirier, E. Sarraute, R. Perrussel (2017), Boundary Element Method for 3D Eddy Current Problems With a Conductive Thin Layer. NUMELEC 2017. https://numelec2017.sciencesconf.org/.

Thanh-Trung Nguyen, Gérard Meunier, Jean-Michel Guichon, and Olivier Chadebec (2015), 3- D Integral Formulation Using Facet Elements for Thin Conductive Shells Coupled With an External Circuit. IEEE Transactions on Magnetics, Vol. 51, Issue 3.

R. Hiptmair and J. Ostrowski (2005), Coupled boundary-element scheme for eddy-current computation, Journal of Engineering Mathematics 51:3, 231-250.

Ana Alonso Rodriguez, and Alberto Valli (2010), Eddy Current Approximation of Maxwell Equations: Theory, Algorithms and Applications, Vol. 4, Springer Science and Business Media.

A. A. Kolyshkin and R. Vaillancourt (1999), Series solution of an eddy-current problem for a sphere with varying conductivity and permeability profiles, IEEE Transactions on Magnetics, vol. 35, no. 6, pp. 44454451.

J.M. Schneider and S.J. Salon (1980), A boundary integral formulation of the eddy-current problem, IEEE Transactions on Magnetics, Vol. MAG-16, No. 5, pp. 1086-1088.

M. Morjaria, S. Mukherjee, F.C. Moons (1982), A boundary integral method for eddy current flow around cracks in thin plates, IEEE Trans. on Magnetics, vol. Mag-18, no 2, p. 467.

K. Schmidt and A. Chernov (2014), Robust transmission conditions of high order for thing conducting sheets in two dimensions, IEEE Trans. Magn., 50(2):41-44.

K. Schmidt and A. Chernov (2013), A unified analysis of transmission conditions for thin conducting sheets in the time-harmonic eddy current model, SIAM J. Appl. Math., 73(6):1980-2003.

T. Le-Duc, G. Meunier, O. Chadebec and J. -M. Guichon (2012), A New Integral Formulation for Eddy Current Computation in Thin Conductive Shells, in IEEE Transactions on Magnetics, vol. 48, no. 2, pp. 427-430.

R. Perrussel and C. Poignard (2013), Asymptotic expansion of steady-state potential in a high contrast medium with a thin resistive layer, Applied Mathematics and Computation, 221:48- 65. 
C. Poignard, P. Dular, R. Perrussel, L. Krähenbühl, L. Nicolas, and M. Schatzman (2008), Approximate conditions replacing thin layer, IEEE Trans. on Mag., 44(6):1154-1157.

S. Rjasanow, O. Steinbach (2007), The fast solution of boundary integral equations, Mathematical and Analytical Techniques with Applications to Engineering, Springer, New York.

F. I. Hantila, I. R. Ciric, A. Moraru, and M. Maricaru (2009), Modelling eddy currents in thin shields, COMPEL, Int. J. Comput. Math. Elect. Electron. Eng., vol. 28, no. 4, pp. 964-973.

\section{Appendix}

\subsection{Calculation of the External Field}

The representation formula of any vector field $E(x)$ for all $x \in \Omega_{ \pm}$:

$$
E(x)=\Psi_{M}\left(\left[\gamma_{D} E\right]_{\Gamma}\right)(x)+\Psi_{A}\left(\left[\gamma_{N} E\right]_{\Gamma}\right)(x)-\operatorname{grad} \Psi_{V}\left(\left[\gamma_{n} E\right]_{\Gamma}\right)(x) .
$$

However

$$
H(x)=-\left(i \omega \mu_{0}\right)^{-1} \operatorname{curl} E(x)
$$

then

$$
\begin{aligned}
H(x) & =-\left(i \omega \mu_{0}\right)^{-1}\left(\operatorname{curlcurl} \int_{\Gamma}\left(n \times\left[\gamma_{D} E\right]_{\Gamma}\right) G(x, y) d S(y)+\operatorname{curl} \int_{\Gamma}\left[\gamma_{N} E\right]_{\Gamma} G(x, y) d S(y)\right), \\
& =-\left(i \omega \mu_{0}\right)^{-1}\left(\int_{\Gamma}-\operatorname{curl}_{\Gamma}\left(\left[\gamma_{D} E\right]_{\Gamma}\right) \nabla_{x} G(x, y) d S(y)+\int_{\Gamma}\left(\nabla_{x} G(x, y) \times\left[\gamma_{N} E\right]_{\Gamma}\right) d S(y)\right) .
\end{aligned}
$$

\subsection{Analytical Solution of a 3D Eddy-Current Problem for a Sphere with a Thin Layer}

Consider a thin layer made of an inner sphere of radius $r_{2}$ and an outer spherical shell of radius $r_{1}$. We introduce the spherical coordinates system $(r, \theta, \phi)$ with center at $O$, where $\theta$ and $\phi$ stands for the azimuthal and polar angle respectively.

We consider the three regions $R_{0}, R_{1}$, and $R_{2}$ defined as follows:

$R_{0}:\left\{r>r_{1}, 0 \leq \theta \leq 2 \pi, 0 \leq \phi \leq \pi\right\}$, containing air,

$R_{1}:\left\{r_{2}<r<r_{1}, 0 \leq \theta \leq 2 \pi, 0 \leq \phi \leq \pi\right\}$, conducting medium where $\sigma$ ans $\mu$ are constants,

$R_{2}:\left\{0 \leq r<r_{2}, 0 \leq \theta \leq 2 \pi, 0 \leq \phi \leq \pi\right\}$, non-conducting medium.

We consider the vector potential formulation with a source term excited by a uniform magnetic field $H_{0}^{s}=$ $1 \vec{z} . H_{0}^{s}$ can be represented by the magnetic vector potential $A_{s}$

$$
A_{s}=\frac{\mu}{2} r \sin (\phi) \vec{\theta}
$$

satisfying

$$
\Delta A_{s}+k^{2} A_{s}=-\mu J_{0}
$$

where $k^{2}=-i \sigma \omega \mu$. 
Using the fact that $A$ has only one non-zero component in $\theta$ direction due to the axial symmetry, we obtain in spherical coordinates

$$
\frac{\partial^{2} A}{\partial r^{2}}+\frac{2}{r} \frac{\partial A}{\partial r}+\frac{\cot \phi}{r^{2}} \frac{\partial A}{\partial \phi}+\frac{1}{r^{2}} \frac{\partial^{2} A}{\partial \phi^{2}}-\frac{A}{r^{2} \sin ^{2} \phi}+k^{2} A=-\mu_{0} J_{0}
$$

with the transmission conditions:

$$
\begin{gathered}
A_{0_{\mid r=r_{1}}}=A_{1_{\mid r=r_{1}}}, \quad A_{1_{\mid r=r_{2}}}=A_{2_{\mid r=r_{2}}}, \\
\left.\frac{\partial A_{0}}{\partial r}\right|_{\mid r=r_{1}}=\left.\frac{\partial A_{1}}{\partial r}\right|_{\mid r=r_{1}},\left.\frac{\partial A_{1}}{\partial r}\right|_{\mid r=r_{2}}=\left.\frac{\partial A_{2}}{\partial r}\right|_{\mid r=r_{2}},
\end{gathered}
$$

where $A_{i}(r, \phi)$ denotes the $\theta$ component of the vector potential in region $R_{i}$, for $i=0 . .2$.

We introduce the following integral transform:

$$
\bar{A}_{i}(r, n)=\frac{1}{D_{n}} \int_{-1}^{-1} A_{i}(r, t) P_{n}^{(1)}(t) d t
$$

where $t=\cos \phi, P_{n}^{(1)}(t)$ is an associated Legendre function of first kind, and

$$
D_{n}:=\int_{-1}^{-1}\left(P_{n}^{(1)}(t)\right)^{2} d t=\frac{2 n(n+1)}{2 n+1} .
$$

Replacing the magnetic properties in (6.2), using this integral transform (6.5), and the fact that $A_{0}=A_{0}^{r}+A_{s}$, we obtain

$$
\begin{gathered}
\frac{\partial^{2} \overline{A_{0}^{r}}}{\partial r^{2}}+\frac{2}{r} \frac{\partial \overline{A_{0}^{r}}}{\partial r}-\frac{n(n+1)}{r^{2}} \overline{A_{0}^{r}}=0, \\
\frac{\partial^{2} \overline{A_{1}}}{\partial r^{2}}+\frac{2}{r} \frac{\partial \overline{A_{1}}}{\partial r}-\frac{n(n+1)}{r^{2}} \overline{A_{1}}+k^{2} \overline{A_{1}}=0, \\
\frac{\partial^{2} \overline{A_{2}}}{\partial r^{2}}+\frac{2}{r} \frac{\partial \overline{A_{2}}}{\partial r}-\frac{n(n+1)}{r^{2}} \overline{A_{2}}=0 .
\end{gathered}
$$

The general solution of (6.6) is:

$$
\overline{A_{0}^{r}}=C_{1} r^{-1-n}+C_{1}^{\prime} r^{n}
$$

but $A_{0}^{r}$ should vanish as $r \rightarrow \infty$, so

$$
\overline{A_{0}^{r}}=C_{1} r^{-1-n} .
$$

The general solution of (6.7) is expressed in terms of Bessel functions:

$$
\overline{A_{1}}=C_{2} r^{-1 / 2} J_{n+\frac{1}{2}}(k r)+C_{3} r^{-1 / 2} Y_{n+\frac{1}{2}}(k r) .
$$

The general solution of (6.8) is:

$$
\overline{A_{2}}=C_{4} r^{n}+C_{4}^{\prime} r^{-1-n}
$$


but the solution must remain bounded as $r \rightarrow 0$, so

$$
\overline{A_{2}}=C_{4} r^{n} .
$$

Inverting the integral transform (6.5), we obtain the solution to the problem in the form

$$
A_{i}(r, \phi)=\sum_{n=1}^{\infty} \bar{A}_{i}(r, n) P_{n}^{(1)}(\cos \phi) \quad i=1 . .2 .
$$

The $\sin \phi$ dependence of the excitation source requires that only $n=1$ be present, with

$$
P_{1}^{(1)}(\cos \phi)=\sin \phi .
$$

Therefore, the solution for the vector potential is

$$
A_{\theta}(r, \phi)=\left\{\begin{array}{lll}
A_{0}(r, \phi)=C_{1} r^{-2} \sin \phi+\frac{\mu}{2} r \sin \phi & R_{0}, \\
A_{1}(r, \phi)=\left(C_{2} r^{-1 / 2} J_{\frac{3}{2}}(k r)+C_{3} r^{-1 / 2} Y_{\frac{3}{2}}(k r)\right) \sin \phi & R_{1}, \\
A_{2}(r, \phi)=C_{4} r \sin \phi & R_{2} .
\end{array}\right.
$$

Using the boundary conditions (6.3)-(6.4), we can determine the constants $C_{i}$ for $i=1 . .4$. 